\title{
Malariometric Indices among Nigerian Children in a Rural Setting
}

\author{
Ekong E. Udoh, ${ }^{1,2}$ Angela E. Oyo-ita, ${ }^{2,3}$ Friday A. Odey, ${ }^{1,2}$ Komomo I. Eyong, \\ Chioma M. Oringanje, ${ }^{2}$ Olabisi A. Oduwole, ${ }^{2}$ Joseph U. Okebe, ${ }^{4}$ Ekpereonne B. Esu, ${ }^{2}$ \\ Martin M. Meremikwu, ${ }^{1,2}$ and Asindi A. Asindi ${ }^{1}$ \\ ${ }^{1}$ Department of Paediatrics, University of Calabar Teaching Hospital, PMB 1278, Calabar, Nigeria \\ ${ }^{2}$ Calabar Institute of Tropical Disease Research and Prevention, University of Calabar Teaching Hospital, P.O. Box 1211, Calabar, Nigeria \\ ${ }^{3}$ Department of Community Medicine, University of Calabar Teaching Hospital, PMB 1278, Calabar, Nigeria \\ ${ }^{4}$ Medical Research Council (UK), Fajara, P.O. Box 273, Banjul, Gambia
}

Correspondence should be addressed to Ekong E. Udoh; rejoicerejoice2001@yahoo.com

Received 12 November 2012; Accepted 8 February 2013

Academic Editor: Neena Valecha

Copyright (C) 2013 Ekong E. Udoh et al. This is an open access article distributed under the Creative Commons Attribution License, which permits unrestricted use, distribution, and reproduction in any medium, provided the original work is properly cited.

\begin{abstract}
Malaria contributes to high childhood morbidity and mortality in Nigeria. To determine its endemicity in a rural farming community in the south-south of Nigeria, the following malariometric indices, namely, malaria parasitaemia, spleen rates, and anaemia were evaluated in children aged 2-10 years. This was a descriptive cross-sectional survey among school-age children residing in a rubber plantation settlement. The children were selected from six primary schools using a multistaged stratified cluster sampling technique. They were all examined for pallor, enlarged spleen, or liver among other clinical parameters and had blood films for malaria parasites. Of the 461 children recruited, $329(71.4 \%)$ had malaria parasites. The prevalence of malaria parasitaemia was slightly higher in the under fives than that of those $\geq 5$ years, $76.2 \%$ and $70.3 \%$, respectively. Splenic enlargement was present in 133 children $(28.9 \%)$. The overall prevalence of anaemia was $35.7 \%$. Anaemia was more common in the under-fives (48.8\%) than in those $\geq 5$ years $(32.8 \%)$. The odds of anaemia in the under fives were significantly higher than the odds of those $\geq 5$ years (OR $=1.95$ [1.19-3.18]). Malaria is highly endemic in this farming community and calls for intensification of control interventions in the area with special attention to school-age children.
\end{abstract}

\section{Introduction}

Malaria remains a leading cause of illness and death in sub-Saharan Africa with the greatest risk seen in children under the age of five, pregnant women, and people living with HIV/AIDS $[1,2]$. About $50 \%$ of Nigerians are estimated to have at least one episode of the disease each year with over 200,000 deaths in children annually [3]. Estimates of malaria burden are based on malariometric indices like prevalence of malaria parasitaemia, spleen rate, and anaemia in defined risk groups [2]. School-age children are vulnerable to the disease and have been studied over the years to determine malaria burden at community levels using these malariometric indices [4].

An understanding of the malaria burden in a given setting is important for health planning, policy development, and control interventions. This study aimed at determining the malaria burden at Ikot-Omin, a rural rubber plantation settlement, using malaria parasitaemia, spleen rate and anaemia as malariometric indices.

\section{Methods}

As part of site preparation for the setting up of a sentinel site for monitoring antimalarial efficacy and drug resistance [5], we conducted a cross-sectional study in Ikot-Omin; a suburb of Calabar in Cross River state, Southern Nigeria. The area is a rubber plantation settlement located 20 kilometres from Calabar, the capital city. It is located at the tropical rain forest belt with average temperature and relative humidity of $28.0^{\circ} \mathrm{C}$ and $80.5 \%$, respectively. The main malaria vectors in the area are Anopheles gambiae and Anopheles funestus. Parasite 
TABLE 1: Prevalence of malariometric indices in children.

\begin{tabular}{|c|c|c|c|c|c|c|c|c|c|}
\hline \multirow{2}{*}{$\begin{array}{l}\text { Age } \\
\text { (years) }\end{array}$} & \multicolumn{3}{|c|}{ Malaria parasitaemia $^{\mathrm{a}}$} & \multicolumn{3}{|c|}{ Splenomegaly $^{\mathrm{b}}$} & \multicolumn{3}{|c|}{ Anaemia $^{c}$} \\
\hline & Present \% & Absent & OR [95\% CI] & Present \% & Absent & OR [95\% CI] & Present \% & Absent & OR [95\% CI] \\
\hline$<5$ & $64(76.4)$ & 20 & $1.35[0.78-2.34]$ & $26(31.0)$ & 58 & 1.13 [0.68-1.89] & $39(48.8)$ & 41 & $1.95[1.19-3.18]$ \\
\hline$\geq 5-10^{*}$ & $265(70.3)$ & 112 & & $107(28.4)$ & 270 & & $119(31.6)$ & 244 & \\
\hline Both age groups & $329(71.4)$ & 132 & & $133(28.9)$ & 328 & & $158(35.7)$ & 285 & \\
\hline
\end{tabular}

${ }^{\mathrm{a}} \chi^{2}=1.17 ; P=0.279$ (not statistically significant).

${ }^{\mathrm{b}} \chi^{2}=0.22 ; P=0.638$ (not statistically significant).

${ }^{c} \chi^{2}=7.28 ; P=0.007$ (statistically significant).

* Baseline age group for calculation of OR [ $95 \% \mathrm{CI}]$.

Prevalence of anaemia was based on 443 children.

resistance to chloroquine and sulfadoxine/pyrimethamine was over $80 \%[6,7]$. The survey was conducted in January 2008.

Children aged 2-10 years attending primary schools in the area were considered eligible for inclusion in the study. They were selected using a multistage stratified cluster sampling technique. The sample size in the study was calculated based on prevalence of malaria parasitaemia in a previous study in a similar setting [8]. Ethical clearance was obtained from the Health Research Ethics Committee of the University of Calabar Teaching Hospital. Children were recruited into the study after parental informed consent had been obtained. A general examination was done, and anthropometric measurements: weight, height, mid-arm circumference (for children between 2 and 5 years), and temperature were taken. Assessment for spleen enlargement was done in all the children following standard methods [9]. Thick and thin blood films were prepared for malaria microscopy and stained using Giemsa. Two microscopists independently read each slide for parasite until 200 white blood cells (WBC) were counted. Discrepancies in the parasite count were resolved by a third microscopist. Parasite density was determined by dividing the parasite count by 200 and multiplying by 8,000 (approximate number of WBCs/microliter) [10]. Blood was also sampled for packed cell volume (PCV). Children with malaria parasitaemia were treated with an appropriate dose of either artemether/lumefantrine or artesunate/amodiaquine combination based on the country's national treatment policy for uncomplicated malaria [11].

The data was double entered and analyzed using Epi-Info version 3.3.2 [12]. The prevalence of malaria parasitaemia, spleen rate, and anaemia $(\mathrm{PCV}<30 \%)$ was calculated as a proportion of children with those indices. Chi square was used for association between categorical variables, while student's $t$-test was used in the test of significance for the difference between two means.

\section{Results}

A total of 461 children participated in the study. The PCV of 18 of them $(3.9 \%)$ could not be obtained either due to breakage of capillary tubes or spillage of blood sample during centrifugation. These were excluded from analysis related to anaemia and mean haematocrit estimation. Two hundred and twenty-nine were males (49.7\%). Eighty-four (18.2\%) were
TABLE 2: Comparison of mean haematocrit values of parasitaemic and aparasitaemic children.

\begin{tabular}{lcccc}
\hline Mean Hct value $(\%)$ & $\mathrm{Mp}+\mathrm{ve}$ & $\mathrm{Mp}-\mathrm{ve}$ & $t$-test & $P$-value \\
\hline Age $<5$ years & $32.5 \pm 4.6$ & $30.7 \pm 7.1$ & 1.33 & 0.19 \\
Age 5-10 years & $34.5 \pm 4.0$ & $34.7 \pm 5.2$ & 0.40 & 0.69 \\
\hline Both age groups & $34.09 \pm 4.21$ & $34.1 \pm 5.7$ & 0.02 & 0.98 \\
\hline
\end{tabular}

under fives, while the rest were aged 5-10 years. The mean age of the study population was $6.48 \pm 2.1$ years.

A positive film was reported in 329 (71.4\%). All had $P$. falciparum monoinfection. The prevalence of parasitaemia was slightly higher in the under fives (76.2\%) compared with those of 5-10 years of age (70.3\%). The spleen rate was $28.9 \%$. There was no significant difference in spleen rate between the under fives (31.0\%) and those of $5-10$ years of age $(28.4 \%)$. The prevalence of anaemia was $48.8 \%$ in the under fives, $32.8 \%$ in those of 5-10 years of age, and $35.7 \%$ for both age categories. The odds of malaria parasitaemia or splenomegaly between the under-fives and those of five years and above were not significantly different. However, the odds of anaemia in the under fives was significantly higher than those of five years and above (OR = 1.95 [1.19-3.18]) (Table 1).

There was no significant difference in the mean haematocrit value between the parasitaemic and aparasitaemic children $(t$ test $=0.02 ; P$ value $=0.98)$ as shown in Table 2 .

\section{Discussion}

The overall prevalence of malaria parasitaemia in the study population was $71.4 \%$. The prevalence of malaria parasitaemia was slightly higher in the under fives compared with those aged 5-10 years. This is consistent with the general observation that the under fives are more vulnerable to the disease in areas of high transmission $[13,14]$. Naturally acquired immunity builds up in older children following repeated exposure to the parasite and is manifested by lower parasite densities and fewer clinical malarial episodes than younger children and less exposed under fives [15].

The prevalence of malaria in this study is much higher than the $59.6 \%$ obtained about a decade ago in a community close to where this study was done [8]. Our finding is also higher than $48.3 \%$ recorded by Wang et al. [16] in a multicenter study conducted among school children aged 
6-10 years living in some East and West African countries. $P$. falciparum was the only species of malaria parasite identified in this study against $P$. falciparum and $P$. malariae reported in a previous study in a nearby community [8].

The high prevalence of malaria and $P$. falciparum monoinfection noted in this study is an indication of a worsening malaria situation around the study area which might be indicative of poor access or adherence to effective control measures such as the use of insecticide treated nets (ITNs). The results of a more recent study, National Malaria Indicator Survey (MIS), have shown an improvement in this situation with a national average prevalence of malaria parasitaemia in preschool children of $42 \%$ [17]. The result of the MIS, however, showed that the prevalence of malaria parasitaemia was the highest (50\%) among children from the lowest wealth quintile. The high prevalence reported in our study population could also be partly explained by the fact that the study population was predominantly poor and rural.

The overall spleen rate in this study is $28.9 \%$. The under fives had a marginally higher spleen rate (31\%) compared with those of 5-10 years of age (28.4\%). These findings buttress the fact that under fives have a more pronounced immunological response (including splenic enlargement) to malaria than the older children [14]. The spleen rate in our study is much higher than the $11.7 \%$ obtained by Adeyemo et al. [18] among apparently healthy children, from a rural primary school in south west Nigeria but less than $44 \%$ and $74.5 \%$ reported in Sierra Leone and India, respectively $[19,20]$. The spleen rate is usually high in areas of high malaria transmission and antimalarial treatment failure. Persistent parasitaemia from treatment failure is known to contribute to splenic enlargement, and this was the case in the India study site [20]. With the introduction of ACTs as the first-line treatment for malaria in Nigeria the spleen rate in under-five children is expected to further decline as a prompt access to effective treatment improves.

Anaemia occurred in $35.7 \%$ of the study population with the under fives having a higher prevalence $(48.8 \%)$ compared with children of five years and above $(32.8 \%)$. The under fives were about twice more likely to be anaemic compared with those of five years and above. These observations agree with the fact that this age group is more vulnerable to $P$. falciparum infection than others with severe and potentially fatal complications ensuing [14]. The prevalence of anaemia among under fives in our study was much lower than $72 \%$ obtained in the national MIS [17]. The difference in the definition and method of determining anaemia between the National MIS and our study is partly responsible for the marked disparity in the results. Whereas the National MIS used a HemoCue to estimate haemoglobin concentration and defined anaemia as $\mathrm{Hb}<11.0 \mathrm{~g} / \mathrm{dL}$ in under fives or $\mathrm{Hb}<11.5 \mathrm{~g} / \mathrm{dL}$ in those of 5-11 years, we used a microematocrit centrifuge to estimate the PCV of the children and defined anaemia as PCV $<30 \%$. The prevalence of anaemia in under fives in this study was comparable with similar studies conducted in Ghana [21]. Studies conducted in India and Venezuela recorded higher prevalence of anaemia in children than what was found in our study [22, 23]. Dietary inadequacy involving all nutrients including iron, high intake of tea which tends to inhibit iron absorption, and poverty were the main reasons for anaemia among children in the Indian study, while in the Venezuela, malaria was reported as the main cause of anaemia [22, 23].

This study shows a marked disparity in the malariometric indices assessed in the children. The highest being the prevalence of malaria parasitaemia (71.4\%), followed by anaemia (35\%), and then spleen rate $(28.9 \%)$. The prevalence of malaria parasitaemia may have been influenced by antimalarial drug use in the community, anaemia by helminthic infestation and dietary intake and spleen rate by the extent and duration of exposure to the parasite [24, 25]. Malaria would have been taken to be mesoendemic in our study area if endemicity was assessed by only the spleen rate and anaemia. Such a conclusion would have misinformed policy makers when deciding on the control measures to implement in that area. Since there is variability in the sensitivity of these indices, multiple estimates of endemicity should be assessed, and the parameter with the highest prevalence be used to inform decision on interventions. This study was conducted in January which is a relatively low malaria transmission period in the country [26]. Since there is seasonal variability in malaria transmission in the country, reporting on point prevalence of malaria parasitaemia is acknowledged as a limitation of the study in determining the endemicity of the disease.

There was no statistically significant difference in the mean haematocrit value between parasitaemic and aparasitaemic children in this study, though some studies have reported such a difference between them [14, 23]. The inability to detect a difference in the mean haematocrit value between both categories of children in this study could be due to the low-grade parasitaemia among those with malaria or that factors other than malaria which were not elucidated in this study might have influenced our finding.

\section{Conclusions}

This study shows that malaria remains a major health problem among school-age children residing in agricultural settlements in the south-south of Nigeria. It is therefor necessary to intensify malaria control efforts with special attention given to school-age children residing in at-risk environments.

Further studies on aetiology of anaemia in children using a uniform methodology are necessary for the development of effective preventive and therapeutic interventions for anaemia in school-age children residing in such environments.

\section{Conflict of Interests}

The authors declare that they have no conflict of interests.

\section{Acknowledgments}

The staff of the selected schools helped in organizing the pupils during the study. Dr. Juan Ikobah and Dr. Nchiwe Ani assisted in the clinical evaluation of the children, while Mr. Bassey Asuquo and the late Mr. Ime Mkpang carefully 
prepared and read the slides for malaria parasite. The authors are very grateful for their contributions to the study.

\section{References}

[1] E. Samba, "Malaria and control efforts in Africa," WHO Newsletter, pp. 9-15, 2000.

[2] World Health Organization, World Malaria Report 2005, WHO, Geneva, Switzerland, 2005.

[3] B. M. Afolabi, E. N. U. Ezedinachi, and B. S. Fatumbi, "Roll back malaria: the story so far," WHO Newsletter, vol. 16, pp. 6-7, 2001.

[4] B. Maegraith, "Malaria," in Adams \& Maegraith Clinical Tropical Diseases, B. Maegraith, Ed., pp. 234-281, Blackwell, Oxford, UK, 6th edition, 1976.

[5] World Health Organization, Assessment and Monitoring of AntiMalarial Drug Efficacy for the Treatment of Uncomplicated Falciparum Malaria, WHO, Geneva, Switzerland, 2003.

[6] A. A. Alaribe, G. C. Ejezie, and E. N. U. Ezedinachi, "Studies on mosquito distribution in Ekemkpon village Cross River State of Nigeria," Journal of Medical Laboratory Sciences, vol. 2, pp. 4551, 2002.

[7] E. N. U. Ezedinachi, A. A. A. Alaribe, M. Meremikwu, and G. C. Ejezie, "New trends in chloroquine efficacy in the treatment of malaria: significance of low (scanty) parasitaemia in an endemic area, with emerging chloroquine-resistant $P$. falciparum," Central African Journal of Medicine, vol. 38, no. 7, pp. 303-307, 1992.

[8] A. A. Alaribe, Dynamics of malaria transmission in Ekemkpon village in Odukpani local government area of Cross River State [Ph.D. thesis], Graduate School, University of Calabar, Calabar, Nigeria, 1999.

[9] J. C. Yang, L. S. Rickman, and S. K. Bosser, "The clinical diagnosis of splenomegaly," Western Journal of Medicine, vol. 155, no. 1, pp. 47-52, 1991.

[10] H. L. Kamga, S. A. Akuro, and A. L. Njunda, "Relationship between blood cell counts and the density of malaria parasites among patients at the regional hospital, Limbe, Cameroun," African Journal of Clinical and Experimental Microbiobiology, vol. 11, no. 2, pp. 120-137, 2010.

[11] Federal Ministry of Health, National Antimalarial Treatment Policy Abuja, Federal Ministry of Health, 2005.

[12] Epi infoTM, Centers for Disease Control and Prevention, Atlanta, Ga, USA.

[13] H. M. Gilles, "Immunology of Malaria," in Bruce-Chwatt's Essential Malariology, H. M. Gilles and D. A. Warrell, Eds., pp. 85-103, Edward Arnold, London, UK, 1993.

[14] T. Chongsuphajaisiddhi, "Malaria," in Diseases in Children in the Sub-Tropics and Tropics, S. Paget, B. Martin, C. Michael, and W. Tony, Eds., pp. 657-674, Edward Arnold, London, UK, 4th edition, 1991.

[15] S. K. Sharma, R. Chattopadhyay, K. Chakrabarti et al., "Epidemiology of malaria transmission and development of natural immunity in a malaria-endemic village, San Dulakudar, in Orissa state, India," The American Journal of Tropical Medicine and Hygiene, vol. 71, no. 4, pp. 457-465, 2004.

[16] S. J. Wang, C. Lengeler, T. A. Smith et al., "Rapid urban malaria appraisal (RUMA) in sub-Saharan Africa," Malaria Journal, vol. 4, article 40, 2005.

[17] National Population Commission (NPC) [Nigeria], National Malaria Control Programme (NMCP) [Nigeria], and ICF International, Nigeria Malaria Indicator Survey 2010: Final Report,
National Population Commission, National Malaria Control Programme, ICF International, 2012.

[18] A. A. Adeyemo, P. E. Olumese, O. K. Amodu, and R. A. Gbadegesin, "Correlates of hepatomegaly and splenomegaly among Healthy school children in a malaria endemic village," Nigerian Journal of Paediatrics, vol. 26, pp. 1-3, 1999.

[19] G. Barnish, G. H. Maude, M. J. Bockarie, O. A. Erunkulu, M. S. Dumbuya, and B. M. Greenwood, "Malaria in a rural area of Sierra Leone. II. Parasitological and related results from preand post-rains clinical surveys," Annals of Tropical Medicine and Parasitology, vol. 87, no. 2, pp. 137-148, 1993.

[20] N. Singh, A. K. Mishra, M. M. Shukla, and S. K. Chand, "Forest malaria in Chhindwara, Madhya Pradesh, central India: a case study in a tribal community," The American Journal of Tropical Medicine and Hygiene, vol. 68, no. 5, pp. 602-607, 2003.

[21] E. Klinkenberg, P. J. McCall, M. D. Wilson et al., "Urban malaria and anaemia in children: a cross-sectional survey in two cities of Ghana," Tropical Medicine and International Health, vol. 11, no. 5, pp. 578-588, 2006.

[22] S. Sharda, K. Kanta, and U. Maijule, "Prevalence of anaemia in Bazigar (Ex-nomadic Tribe) preschool children in Punjab," Journal of Human Ecology, vol. 21, pp. 265-267, 2007.

[23] S. P. Mato, "Anemia and malaria in a Yanomami Amerindian population from the Southern Venezuelan Amazon," The American Journal of Tropical Medicine and Hygiene, vol. 59, no. 6, pp. 998-1001, 1998.

[24] J. French and B. M. Camitta, "Splenomegaly," in Nelson Text Book of Paediatrics, R. E. Behrman, R. M. Kliegman, and H. B. Jenson, Eds., pp. 1675-1676, WB Saunders, Philadelphia, Pa, USA, 17th edition, 2004.

[25] D. J. Bradley, C. I. Newbold, and D. A. Warrell, "Malaria," in Textbook of Medicine, D. J. Weatherall, J. G. G. Ledingham, and D. A. Warrell, Eds., pp. 5.474-5.502, Oxford University Press, Oxford, UK, 2nd edition, 1987.

[26] K. Olayemi, A. T. Ande, A. V. Ayanwale et al., "Seasonal trends in epidemiological and entomological profiles of malaria transmission in North Central Nigeria," Pakistan Journal of Biological Sciences, vol. 14, no. 4, pp. 293-299, 2011. 


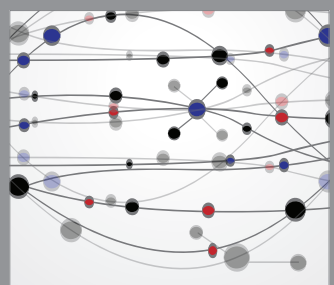

The Scientific World Journal
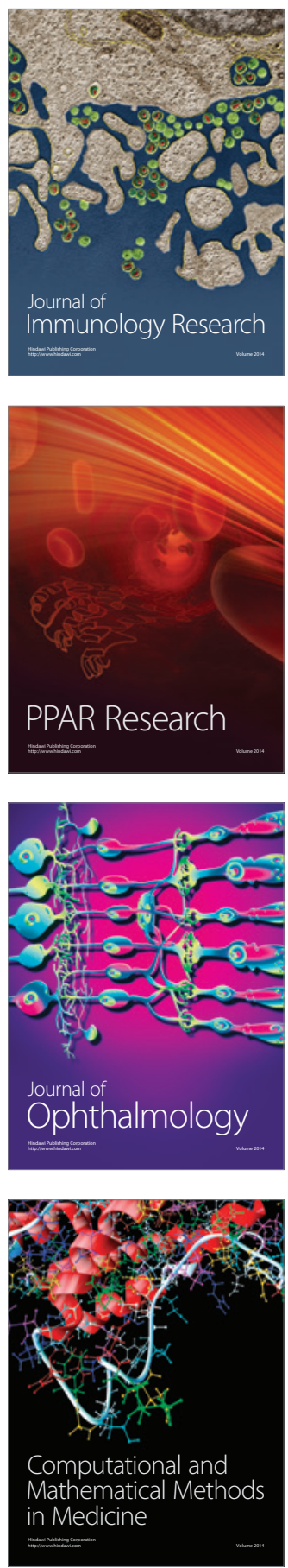

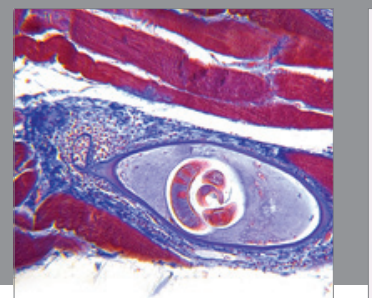

Gastroenterology

Research and Practice
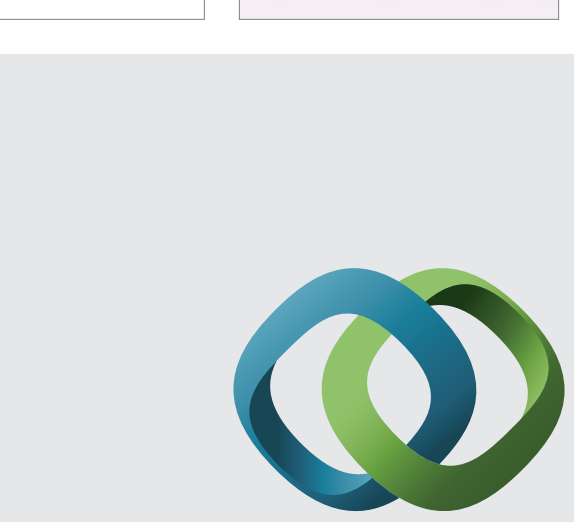

\section{Hindawi}

Submit your manuscripts at

http://www.hindawi.com
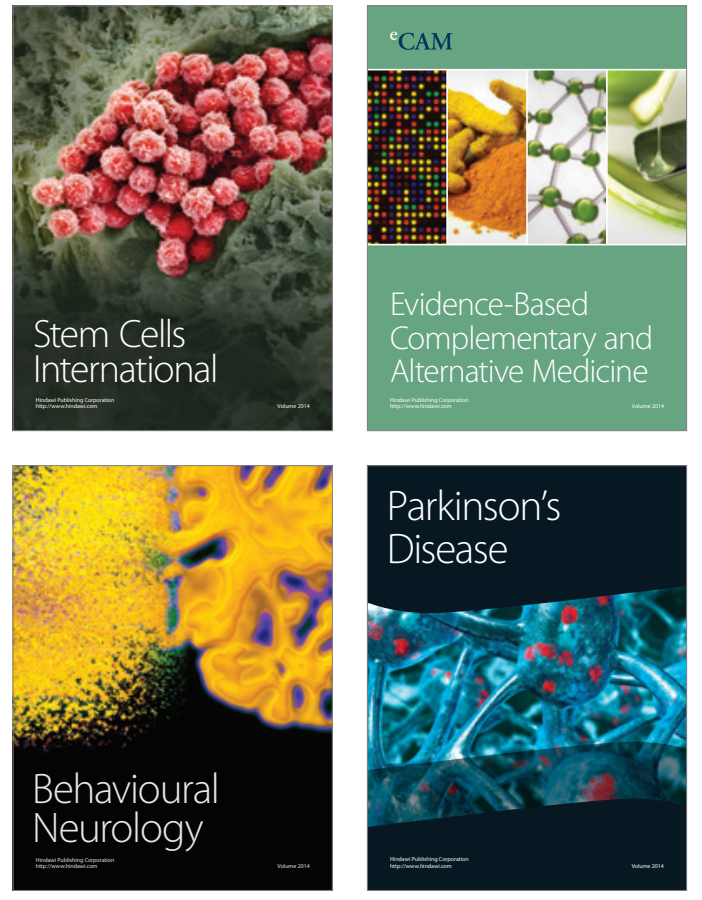
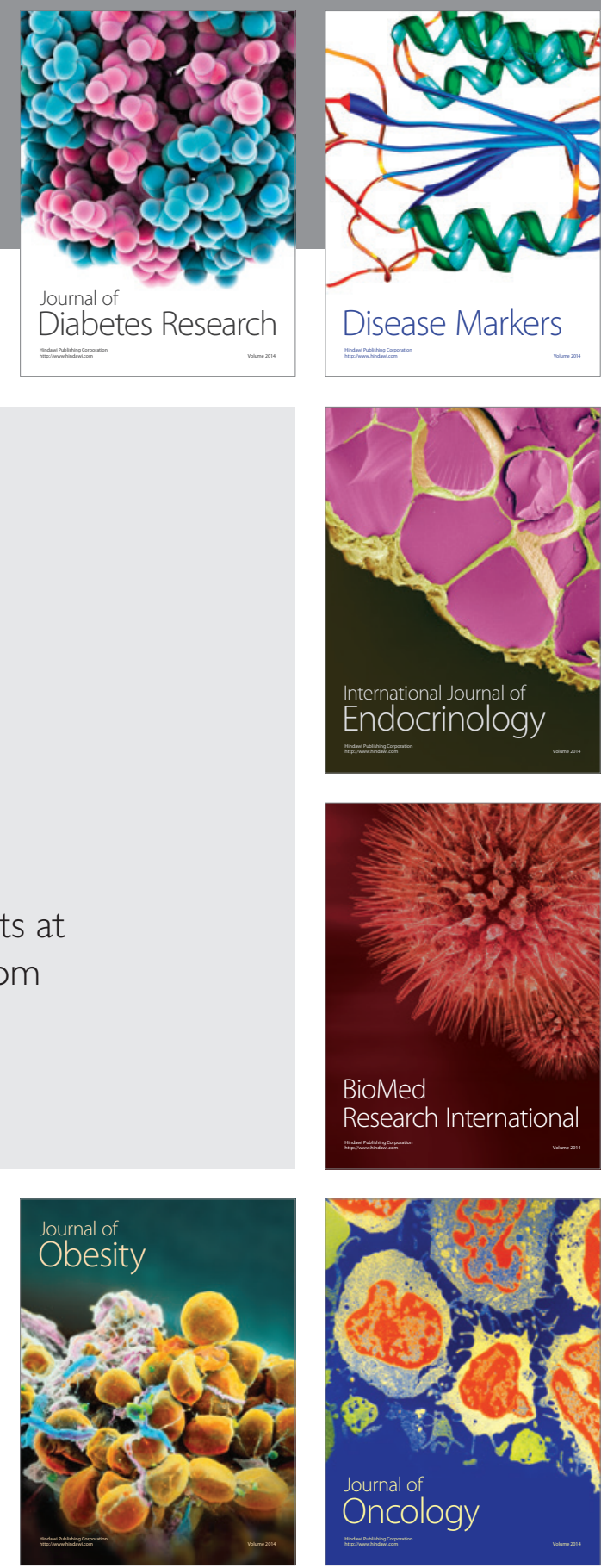

Disease Markers
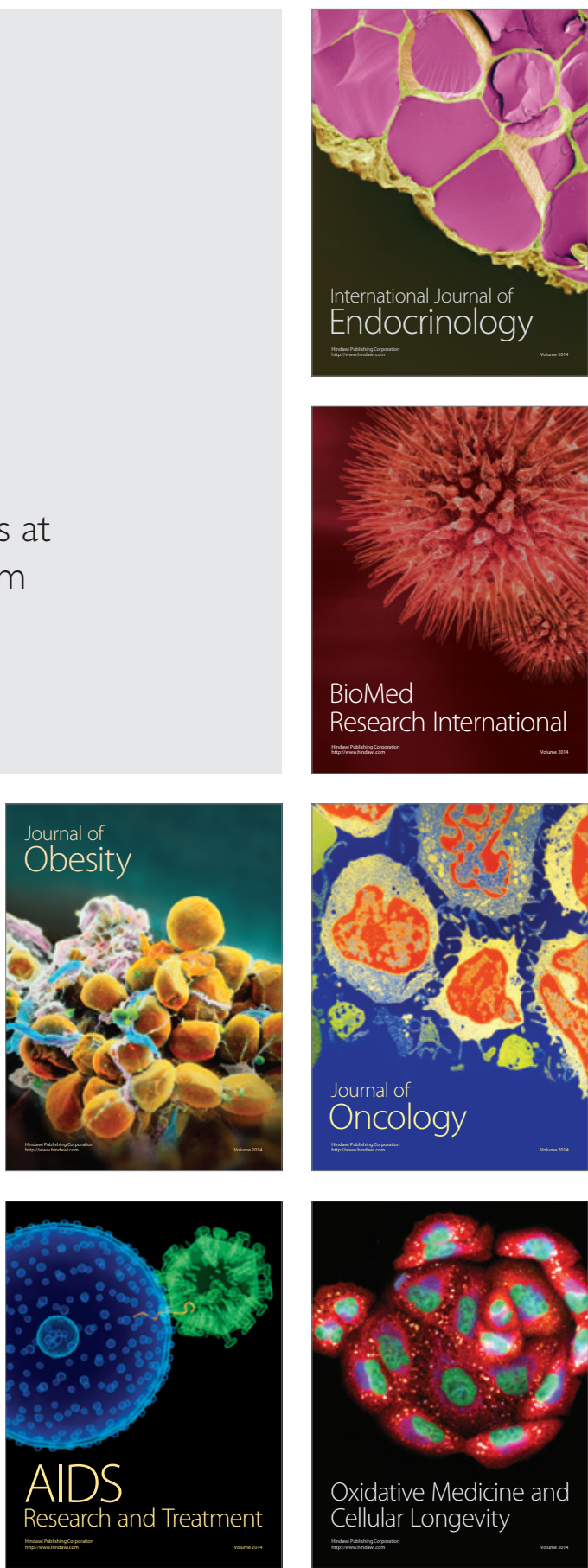